\title{
THE APPLICATION OF A CLASS OF ONE-STEP METHODS TO SOLVE THE INITIAL VALUE PROBLEM
}

A method for the numerical solution of initial value problems with different step sizes is described. A procedure which realizes this method is also given.

1. Introduction. For the numerical solution of the initial value problem for the system of ordinary differential equations

$$
\frac{d y}{d x}=f(x, y), \quad y\left(x_{0}\right)=y_{0}, \quad y, y_{0} \in R^{s}, \quad s \geqslant 1,
$$

we use a one-step method.

The realization of any one-step method may have the following form

$$
\begin{aligned}
\eta_{0} & :=y_{0} \\
\eta_{i+1} & :=\eta_{i}+h_{i} \Phi\left(x_{i}, \eta_{i}, h_{i}\right), \quad i=0,1,2, \ldots, \\
x_{i+1} & :=x_{i}+h_{i},
\end{aligned}
$$

where $\Phi(x, y, h)$ is the increment function of the one-step method and $\eta_{i}, \Phi\left(x_{i}, \eta_{i}, h_{i}\right) \in R^{s}$.

Here, for the numerical solution of the problem (1.1) we use the one-step methods which were given by Bobkov (see Krylov et al. [2]). The Bobkov method is described by the parameters $A_{i}, \alpha_{i}(i=0,1, \ldots, q)$ and some formulae for the approximation of the solutions at knots $\alpha_{i}$. The parameters $A_{i}, \alpha_{i}(i=0,1, \ldots, q)$ are obtained from the condition

$$
y(x+h)-y(x) \approx h \sum_{i=0}^{q} A_{i} y^{\prime}\left(x+\alpha_{i} h\right)=h \sum_{i=0}^{q} A_{i} f_{n+\alpha_{i}},
$$

where equality holds up to the terms with $h^{p}$, and $p$ is the order of the onestep method. In what follows $\tilde{f}_{n+1}=f\left(x+h, \tilde{\eta}_{n+1}\right)$ and $\tilde{\eta}_{n+1}$ is not the final numerical solution of $(1.1)$ at point $x+h$, i.e. for $\tilde{\eta}_{n+1}$ we have $y(x+h)=\tilde{\eta}_{n+1}$ $+O\left(h^{p}\right)$. 
For example, the second order Bobkov method may have the form

$$
\begin{aligned}
& \tilde{\eta}_{n+1}=\eta_{n}+h f_{n}, \\
& \eta_{n+1}=\eta_{n}+(h / 2)\left(f_{n}+\tilde{f}_{n+1}\right),
\end{aligned}
$$

where $A_{0}=A_{1}=\frac{1}{2}$ and $\alpha_{0}=0, \alpha_{1}=1$.

This method is equivalent to the Heun method.

2. A method of step size control. The presented method may be applied to the problem (1.1) with $s>1$, but the formulae below are given for one differential equation only.

From (1.2) we see that the local truncation error of the Bobkov method has the following form

$$
r=h^{p+1} y^{(p+1)}(x)\left[\frac{1}{(p+1) !}-\frac{1}{p !} \sum_{i=0}^{q} A_{i} \alpha_{i}^{p}\right]+O\left(h^{p+2}\right),
$$

where $p$ is the order of the method.

Let

$$
\gamma=\frac{1}{(p+1) !}-\frac{1}{p !} \sum_{i=0}^{q} A_{i} \alpha_{i}^{p}
$$

We see that $\gamma$ depends on the parameters $A_{i}, \alpha_{i}(i=0,1, \ldots, q)$. If we use the one-step method for the numerical solution of (1.1) with the step size $h$, we have

$$
y(x+h)-\eta(x+h, h) \doteq C(x) h^{p+1},
$$

where $\eta(x+h, h)$ is the numerical solution obtained with the step size $h$ and $p$ is the order of this method.

After application of the same one-step method with the step size $h / 2$ we have [1]

$$
y(x+h)-\eta(x+h, h / 2) \doteq 2 C(x)(h / 2)^{p+1} .
$$

In a typical situation the step size control mechanism is made on the basis of the solutions $\eta(x+h, h)$ and $\eta(x+h, h / 2)$. In the paper [1] the following algorithm of the step size control is given

$$
h_{\text {new }}:=h_{\text {old }} / w \text {, }
$$

where

$$
w:=1.25 \sqrt[p+1]{\frac{1}{2\left(2^{p}-1\right)} \max _{1 \leqslant k \leqslant s} \frac{\left|\eta_{k}(x+h, h)-\eta_{k}(x+h, h / 2)\right|}{\left|\eta_{k}^{*}(x+h)\right| \cdot e p s}}
$$

and

$$
\eta^{*}(x+h):=\eta(x+h, h / 2)+\frac{\eta(x+h, h / 2)-\eta(x+h, h)}{2^{p}-1} .
$$


The constant 1.25 in (2.3) has been chosen experimentally and gives a safe algorithm.

Now we use the same mechanism for the step size control as was described in [1] and is given above in short form. However, we obtain $\eta(x+h, h / 2)$ in a different way. By a special choice of the coefficient $\gamma$ we may simulate calculation. with the step size $h / 2$.

If we have the Bobkov method which satisfies

$$
y(x+h)-\eta_{n+1} \doteq \gamma y^{(p+1)}(x) h^{p+1},
$$

we may find another method of the same order which gives

$$
y(x+h)-\bar{\eta}_{n+1} \doteq 2 \gamma y^{(p+1)}(x)\left(\frac{h}{2}\right)^{p+1}=\frac{\gamma}{2^{p}} y^{(p+1)}(x) h^{p+1} .
$$

The obtained solution $\bar{\eta}_{n+1}$ may be treated as $\eta(x+h, h / 2)$ in $(2.1)$ and the formulae (2.2)-(2.4) may be used with $\eta_{n+1}$ and $\bar{\eta}_{n+1}$.

The solution at knots $x_{i}(i=0,1, \ldots, q)$ must be obtained with local error of order not less than $p$. For example, consider the second order method. We obtain the parameters of the method from the system

$$
\sum_{i=0}^{q} A_{i}=1, \quad \sum_{i=0}^{q} A_{i} \alpha_{i}=\frac{1}{2}
$$

which is formed from (1.2) and also we have the relationship

$$
\sum_{i=0}^{q} A_{i} \alpha_{i}^{2}=\frac{1}{3}-2 \gamma
$$

Let $q=1$ and $\alpha_{0}=0$, then we obtain

$$
\alpha_{0}=0, \quad A_{0}=\frac{1-24 \gamma}{4(1-6 \gamma)}, \quad \alpha_{1}=\frac{2}{3}(1-6 \gamma), \quad A_{1}=\frac{3}{4(1-6 \gamma)} .
$$

To have a one-step character of the method we must take $0<\alpha_{1} \leqslant 1$ and from this restriction we obtain

$$
-1 / 12 \leqslant \gamma<1 / 6
$$

Instead of $\eta_{n+\alpha}$ we use $y_{n+\alpha}$ in the following formulae. Taking $\gamma=-1 / 12$ we have the formula

Now we have to obtain a formula with $\gamma=-\frac{1}{48}$, i.e.

$$
y_{n+1}=y_{n}+(h / 2)\left(f_{n}+f_{n+1}\right) \text {. }
$$

$$
y_{n+1}=y_{n}+(h / 3)\left(f_{n}+2 f_{n+3 / 4}\right) \text {. }
$$

This formula is the same as (2.5) used with the step size $h / 2$ (with respect to the first term of the error). For the calculations of $\tilde{f}_{n+1}, f_{n+3 / 4}$ with order not 
less than 2 we may apply for (2.5) the formulae

$$
\begin{aligned}
y_{n+1 / 2} & =y_{n}+(h / 2) f_{n}, \\
\tilde{y}_{n+1} & =y_{n}+h f_{n+1 / 2},
\end{aligned}
$$

and for (2.6) the formulae

$$
\begin{aligned}
& y_{n+3 / 8}=y_{n}+\frac{3}{8} h f_{n}, \\
& y_{n+3 / 4}=y_{n}+\frac{3}{4} h f_{n+3 / 8} .
\end{aligned}
$$

Of course, we may apply here also other formulae.

In a general situation we may use the formulae

$$
\begin{aligned}
y_{n+\alpha / 4} & =y_{n}+(\alpha / 4) h f_{n}, \\
y_{n+\alpha / 2} & =y_{n}+(\alpha / 2) h f_{n+\alpha / 4} \\
y_{n+\alpha} & =y_{n}+\alpha h f_{n+\alpha / 2}, \\
y_{n+1} & =y_{n}+A_{0} h f_{n}+A_{1} h f_{n+\alpha / 2}
\end{aligned}
$$

and

$$
y_{n+1}=y_{n}+B_{0} h f_{n}+B_{1} h f_{n+\alpha} .
$$

In this paper the procedure diffsysthek (in ALGOL 60) which realizes the formulae (2.7) and (2.8) with $\alpha=1$ is presented. The new step size $h$ is computed from the formulae $(2.2)-(2.4)$. It is not necessary to obtain a solution with $\gamma$ and $\gamma / 2^{p}$, one may also use different values of $\gamma$ and $\gamma_{0}$.

When we know $\gamma$ and $\gamma_{0}$ we may also apply Richardson's extrapolation to obtain a better solution. Heun's method uses formula (2.4). problems

3. Numerical experiments. We have tested our procedure for the
(A) $y_{1}^{\prime}=1 / y_{2}, \quad y_{1}(0)=1, \quad y_{1}=e^{x}$, $y_{2}^{\prime}=-1 / y_{1}, \quad y_{2}(0)=1, \quad y_{2}=e^{-x}$
(B) $y^{\prime}=10 \cos 10 x, \quad y(0)=0, \quad y=\sin 10 x$;
$\begin{array}{lll}y_{1}^{\prime}=10 \operatorname{sgn} \sin (20 x) y_{2}, & y_{1}(0)=0, & y_{1}=|\sin 10 x|, \\ y_{2}^{\prime}=-10 \operatorname{sgn} \sin (20 x) y_{1}, & y_{2}(0)=1, & y_{2}=|\cos 10 x|\end{array}$

The calculations were made for $e p s=e t a={ }_{10}-3,{ }_{10}-6,{ }_{10}-9$ (for) problem (C) only for eps $=10-3$ ) at points $x=0.5,1.0,1.5,10.0$. Tables $1-4$ present the relative error $\left(y_{n}-y(x)\right) / y(x)$ and the number of evaluations of the function $f([f])$ at points $x=1.5$ and $x=10.0$. 
TA B LE 1. Problem (A) (diffsysthek procedure)

\begin{tabular}{rrrrrrr}
\hline$x$ & \multicolumn{1}{c}{$10-3$} & {$[f]$} & \multicolumn{1}{c}{$10-6$} & {$[f]$} & $10-9$ & {$[f]$} \\
\hline 1.5 & $-2.7_{10}-4$ & 4 & $-1.4_{10}-7$ & 31 & $5.1_{10}-11$ & 255 \\
& $2.5_{10}-4$ & & $1.3_{10}-7$ & & $9.7_{10}-11$ & \\
10.0 & $-2.3_{10}-3$ & 54 & $-2.5_{10}-6$ & 442 & $-6.2_{10}-10$ & 4266 \\
& $2.0_{10}-3$ & & $-2.4_{10}-6$ & & $6.6_{10}-10$ & \\
\hline
\end{tabular}

TABLE 2. Problem (A) (Heun method)

\begin{tabular}{rrrrr}
\hline$x$ & \multicolumn{1}{c}{$10^{-3}$} & {$[f]$} & \multicolumn{1}{c}{$10-9$} & {$[f]$} \\
\hline 1.5 & $-6.92_{10}-5$ & 19 & $4.15_{10}-10$ & 1089 \\
& $-4.85_{10}-4$ & & $-1.22_{10}-9$ & \\
10.0 & $1.91_{10}-2$ & 148 & $1.94_{10}-8$ & 13018 \\
& $-2.95_{10}-2$ & & $-2.42_{10}-8$ & \\
\hline
\end{tabular}

TABLE 3. Problem (B) (diffsysthek procedure)

\begin{tabular}{rrrrrrr}
\hline$x$ & \multicolumn{1}{c}{$10^{-3}$} & {$[f]$} & \multicolumn{1}{c}{$10-6$} & {$[f]$} & \multicolumn{1}{c}{$10-9$} & {$[f]$} \\
\hline 1.5 & $-1.3_{10}-4$ & 27 & $1.6_{10}-8$ & 255 & $1.0_{10}-9$ & 2527 \\
10.0 & $1.0_{10}-2$ & 447 & $6.2_{10}-7$ & 4912 & $-2.4_{10}-8$ & 49059 \\
\hline
\end{tabular}

TAB LE 4. Problem (C)

\begin{tabular}{ccccc}
\hline & \multicolumn{3}{c}{ (diffsysthek procedure) } & \multicolumn{2}{c}{ (Heun method) } \\
$x$ & $10^{-3}$ & {$[f]$} & \multicolumn{1}{c}{$10^{-3}$} & {$[f]$} \\
\hline \multirow{2}{*}{1.5} & $-1.3_{10}-1$ & 129 & $-2.64_{10}-3$ & 988 \\
& $8.0_{10}-2$ & & $2.64_{10}-3$ & \\
10.0 & $7.9_{10} 0$ & 1113 & $-1.65_{10}-2$ & 11648 \\
& $4.4_{10}-1$ & & $-1.65_{10}-2$ & \\
\hline
\end{tabular}

4. Conclusions. In the same way as is described in Section 2 we may obtain methods with different values of parameter $\gamma$. We may "simulate" the calculations with different step size sequences, for example with $h / 2, h / 3, \ldots$, but with respect to the first term of the error only.

\section{Description of procedure diffsysthek}

Procedure declaration. The procedure diffsysthek solves the initial value problem of the form

(2)

$$
\begin{gathered}
y_{k}^{\prime}=f_{k}\left(x, y_{1}(x), y_{2}(x), \ldots, y_{n}(x)\right), \\
y_{k}\left(x_{0}\right)=y_{0 k} \quad(k=1,2, \ldots, n)
\end{gathered}
$$

at the points $x_{1}, x_{2}, \ldots$ 
Data:

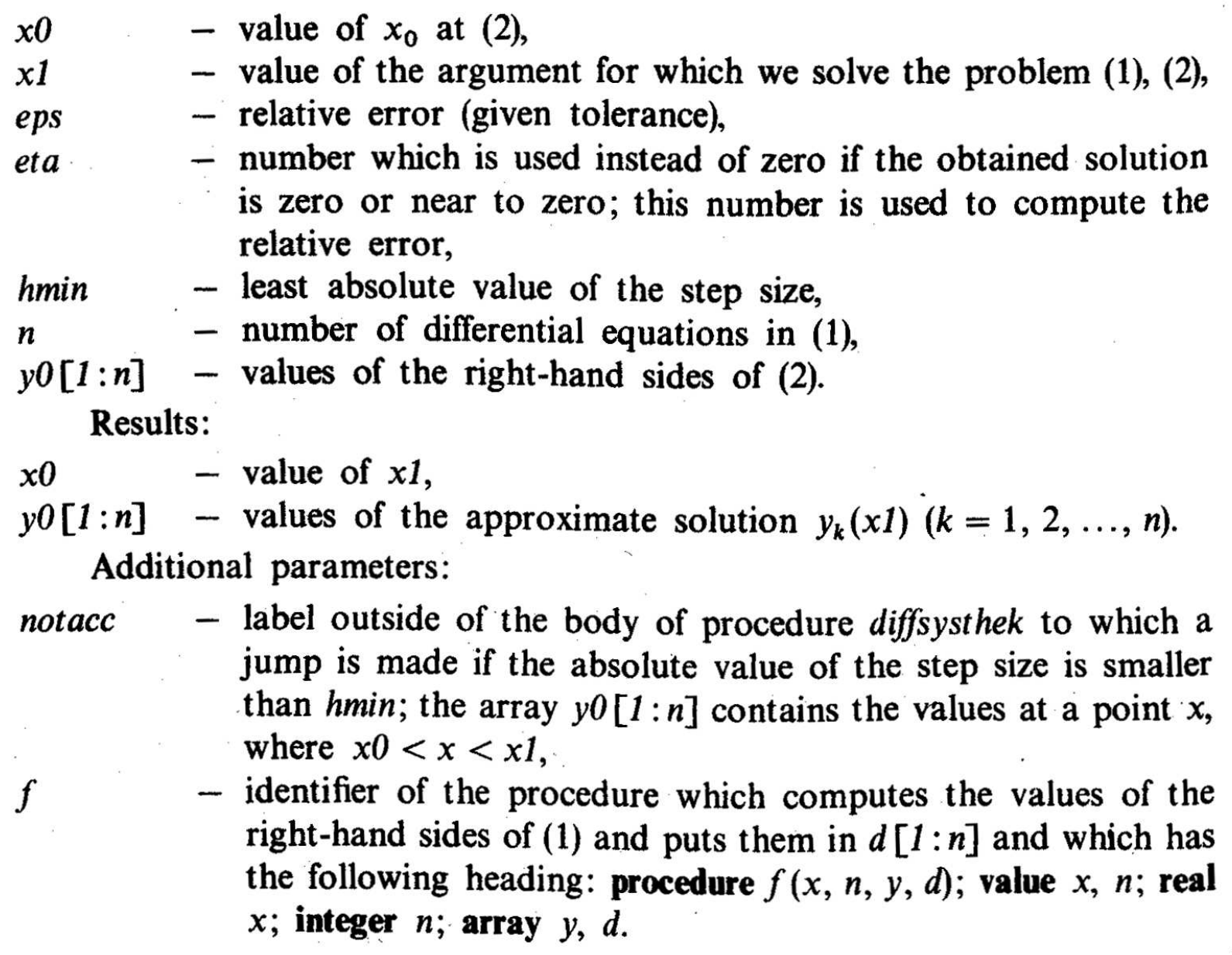

\section{References}

[1] J. Chomicz, A. Olejniczak, M. Szyszkowicz, Dobór kroku obliczeń dla metod jednokrokowych, Raport nr N-35, Instytut Informatyki Uniwersytetu Wrockawskiego, Wroclaw 1978.

[2] V. I. Krylov, V. V. Bobkov and P. I. Monasty rnyĩ, Numerical methods in higher, mathematics (in Russian), Vol. 2, Moscow 1977.

INSTITUTE OF COMPUTER SCIENCE

UNIVERSITY OF WROCEAW

51-151 WROCŁAW

Received on 1981.01.09;

revised version on 1982.03.11 
procedure diffsysthek $(x 0, x 1$, eps, eta, $h$ min, $n, y 0$, notacc, $f)$;

value $\times 1$, eps, eta, $\operatorname{mmin,n}$;

real $\times 0, \times 1$, eps, eta, hmin;

integer n;

array yo;

label notacc;

procedure f;

begin

real h, hh, ww, w3, w4;

integer i;

Boolean last;

array $d, y, y f[1: n]$;

eps : $=.008 /$ eps;

$h:=x 1-\times 0$;

last : $=$ true;

$\left.f\left(\times 0, n, y^{n}\right), y^{f}\right)$;

conth;

$h h:=.25 \times h ;$

for $i:=1$ step 1 until $n$ do

$y[i]:=y 0[i]+h h \times y f[i]$;

$f(x 0+h h, n, y, d)$;

hh: $=h h+h h$;

for $i:=1$ step 1 until $n$ do

$y[i]:=y 0[i]+h h \times d[i]$;

$f(x 0+h h, n, y, d)$;

for $i:=1$ step 1 unt $i$ l $n$ do

$y[i]:=y 0[i]+h \times d[i] ;$

$f(x 0+h, n, y, d)$;

$m w:=.0$; 


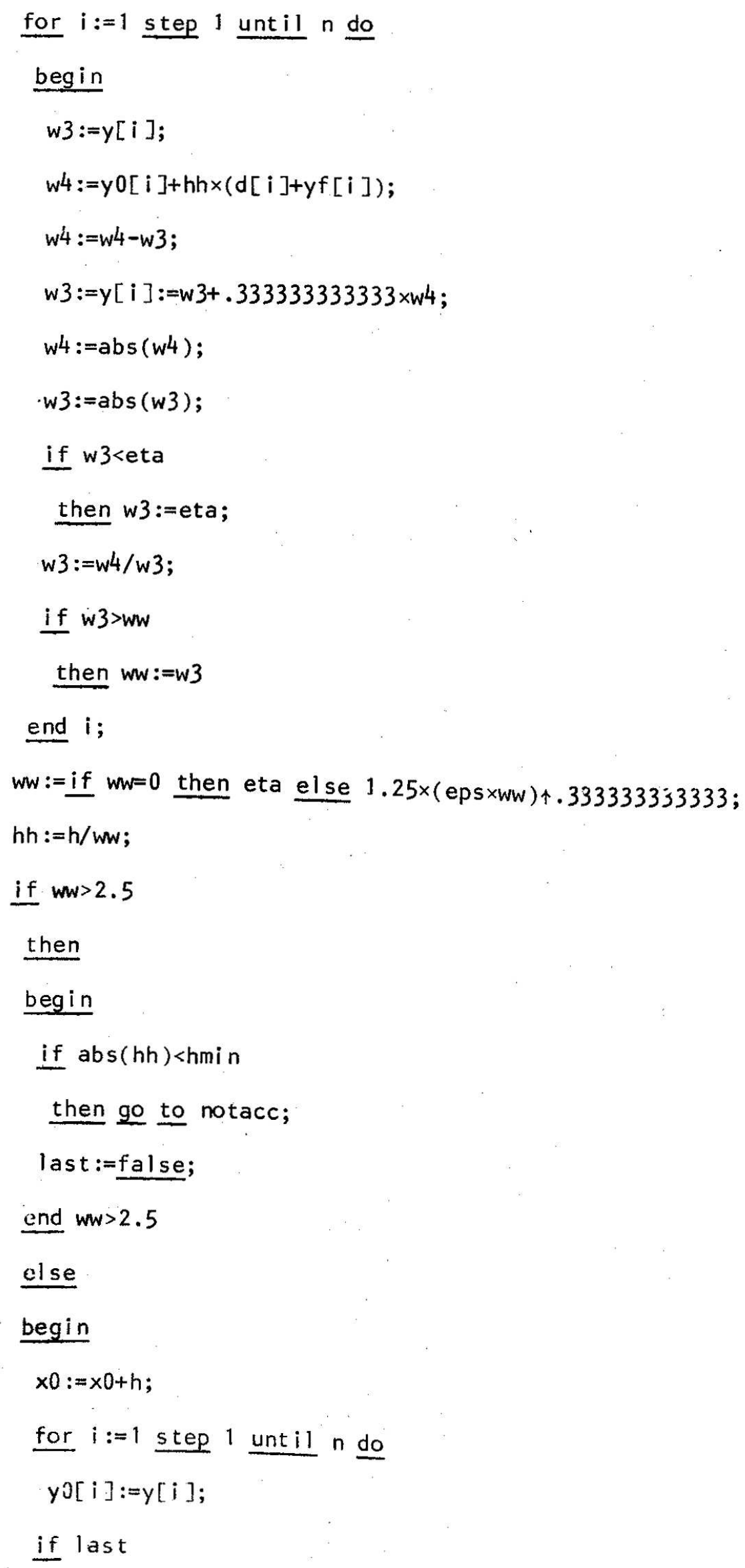


then go to endp;

$f(x 0, n, y 0, y f)$;

w3:=x1-x0;

if $(w 3-h h) \times h<0$

then

begin

hh :=w3;

last $:=$ true

end $(w 3-h h) \times h<0$

end $w w<2.5$;

$h:=h h$;

go to conth;

endp :

end diffsysthek; 